\title{
SCHOENFLIES SOLUTIONS OF CONFORMAL BOUNDARY VALUES MAY FAIL TO BE SOBOLEV
}

\author{
Yi Ru-Ya Zhang \\ Hausdorff Center for Mathematics \\ Endenicher Allee 60, D-53115 Bonn, Germany; yizhang@math.uni-bonn.de
}

\begin{abstract}
There exists a planar Jordan domains $\Omega$ with 1-Hausdorff dimensional boundary such that, for any conformal map $\varphi: \mathbf{D} \rightarrow \Omega$, any homeomorphic extensions to the entire plane of either $\varphi$ or $\varphi^{-1}$ cannot be in $W_{\text {loc }}^{1,1}$ class (or even not in $B V_{\text {loc }}$ ).
\end{abstract}

\section{Introduction}

Let $\Gamma \subset \mathbf{C}$ be a Jordan curve, namely there exists a homeomorphism $\phi: \mathbf{S}^{1} \rightarrow \Gamma$, where $\mathbf{C}$ is the complex plane and $\mathbf{S}^{1}$ denotes the boundary of the unit disk $\mathbf{D}$. According to Jordan curve theorem, the curve $\Gamma$ divides $\mathbf{C}$ into two components, and we call the bounded component a Jordan domain.

Jordan-Schoenflies theorem states that any homeomorphism between two Jordan curves on $\mathbf{C}$ can be extended to a homeomorphism between the entire $\mathbf{C}$; see [12, Corollary 2.9]. To be more precise, given two Jordan domains $\Omega_{1}$ and $\Omega_{2}$ and a homeomorphism $\varphi: \partial \Omega_{1} \rightarrow \partial \Omega_{2}$, there exists a homeomorphism $\Phi$, which we call a Schoenflies solution of the boundary value $\varphi$, from $\mathbf{C}$ to $\mathbf{C}$ such that the restriction of $\Phi$ to $\Gamma_{1}$ coincides with $\varphi$. Then a natural question arises:

Question 1.1. Given two Jordan domains $\Omega_{1}, \Omega_{2} \subset \mathbf{C}$ together with a homeomorphism $\varphi: \partial \Omega_{1} \rightarrow \partial \Omega_{2}$, what is the best regularity of Schoenflies solutions of the boundary value $\varphi$ ?

Certainly the answer to this question depends on the given boundary value and the geometry of both $\Omega_{1}$ and $\Omega_{2}$. Let us recall some known results. If $\Omega_{2}$ is bounded by a smooth Jordan curve, then by the techniques from differential topology for each conformal map we can find a smooth Schoenflies solution to any homeomorphism from $\mathbf{S}^{1}$ onto $\partial \Omega$. Assume that $\varphi: \mathbf{S}^{1} \rightarrow \partial \Omega_{2}$ is quasisymmetric, via Douady-Earle extension theorem there exists a $K$-quasiconformal Schoenflies solution $\Phi$. By [1], we further have that both $\Phi$ and $\Phi^{-1}$ are in $W_{\text {loc }}^{1, p}(\mathbf{C})$ for any $p<2 K /(K-1)$. Recently Koskela, Pankka and the author have been working on a version of this result for domains satisfying Gehring-Martio conditions [8].

Recall Carathéodory's theorem states that, given any two Jordan domain $\Omega_{1}$ and $\Omega_{2}$, every conformal map $\varphi: \Omega_{1} \rightarrow \Omega_{2}$ can be continuously extended to the boundary as a homeomorphism $\varphi: \bar{\Omega}_{1} \rightarrow \bar{\Omega}_{2}$. We abuse $\varphi$ here. In this paper we investigate Question 1.1 with the boundary value given by Carathéodory's theorem, namely a conformal boundary value.

The main result of this paper is the following.

https://doi.org/10.5186/aasfm.2019.4441

2010 Mathematics Subject Classification: Primary 30C70.

Key words: Sobolev homeomorphism, Jordan-Schoenflies theorem.

This work was supported by the Academy of Finland via the Centre of Excellence in Analysis and Dynamics Research (Grant no. 271983) and the Hausdorff Center for Mathematics. 
Theorem 1.2. There exists a Jordan domain $\Omega \subset \hat{\mathbf{C}}$ with 1-Hausdorff dimensional boundary such that, any Schoenflies solution of any conformal boundary value $\varphi: \mathbf{S}^{1} \rightarrow \partial \Omega$ or $\phi: \partial \Omega \rightarrow \mathbf{S}^{1}$ is not in $W_{\text {loc }}^{1,1}(\mathbf{C})$ (even not in $B V_{\text {loc }}(\mathbf{C})$ ).

This result indicates that, in general, one cannot expect the regularity of Schoenflies solutions to a given boundary value to be better than homeomorphism; even if the boundary value is given by a (extended) conformal map (which is a quite natural choice). Thus, geometric assumptions on the Jordan domain in question and (energy) controls on the boundary value are necessary. One can see e.g. [2, 13, 6, 9] for recent results in this direction. Especially in the very recent paper [10] Koski and Onninen give positive answers to Question 1.1 under certain circumstances.

The notation in the paper is quite standard. The Euclidean distance between two sets $A, B \subset \mathbf{R}^{2}$ is denoted by $\operatorname{dist}(A, B)$. We denote by $\ell(\gamma)$ the length of a curve $\gamma$. For a set $A \subset \mathbf{R}^{2}$, we write its boundary as $\partial A$, and its closure as $\bar{A}$, respectively, with respect to the Euclidean topology. We use the notation $\mathcal{H}^{1}$ for 1-dimensional Hausdorff measure.

Acknowledgement. The author would like to express his sincere gratitude to the referee for his (or her) nice review and useful suggestions on this paper. Especially a serious mistake in the previous version was pointed out. The author also would like to thank Professor Jani Onninen for posing this interesting question.

\section{Proof of Theorem 1.2}

Define the inner distance with respect to $\Omega$ between $x, y \in \Omega$ by

$$
\operatorname{dist}_{\Omega}(x, y)=\inf _{\gamma \subset \Omega} \ell(\gamma)
$$

where the infimum runs over all curves joining $x$ and $y$ in $\Omega$.

2.1. Schoenflies solution of conformal boundary value $\varphi: S^{\mathbf{1}} \rightarrow \partial \Omega$. The idea of the proof is that, we construct a Jordan domain $\Omega \subset \hat{\mathbf{C}}$ satisfying that there exists a (Cantor) set $E \subset \partial \Omega$ such that,

(i) for any conformal $\varphi: \mathbf{D} \rightarrow \Omega$, i.e. for any conformal boundary value, we have

$$
\mathcal{H}^{1}\left(\varphi^{-1}(E)\right)>0
$$

(ii) for any point $x$ in the complementary domain $\widetilde{\Omega}$,

$$
\operatorname{dist}_{\widetilde{\Omega}}(x, E \backslash\{(0,0),(1,0)\})=\infty .
$$

If such a Jordan domain exists (see Lemma 2.1 below), then by (i) and (ii), any Schoenflies solution of the conformal boundary value $\varphi$ is not in $W_{\text {loc }}^{1,1}$ (even not in $\left.B V_{\text {loc }}\right)$ by Fubini's theorem; indeed, such a solution maps a family of radial segments in the exterior of the unit disk (with finite length) into a family curves of infinite length in $\widetilde{\Omega}$. By calculating in the polar coordinate we know that such a map cannot be in $W_{\text {loc }}^{1,1}$ (even not in $B V_{\text {loc }}$ ). Hence Theorem 1.2 follows.

We first construct a Jordan curve $\Gamma$ in the plane. Towards this, let us recall the construction of a fat Cantor set $E \subset[0,1]$ on the real axis. Let $C_{0}=I_{0,1}=[0,1]$ and $C_{i}$ with $i \geq 1$ recursively as follows: When $I_{i, j}=[a, b]$ has been defined, let

$$
I_{i+1,2 j-1}=\left[a, \frac{a+b-4^{-i}}{2}\right] \quad \text { and } \quad I_{i+1,2 j}=\left[\frac{a+b+4^{-i}}{2}, b\right]
$$


i.e. we remove an open interval of length $4^{-i}$ from the middle of the interval $I_{i, j}$. Then we set

$$
C_{i}=\bigcup_{j=1}^{2^{i}} I_{i, j}
$$

The set $E$ is finally given by

$$
E=\bigcap_{i=1}^{\infty} C_{i} .
$$

A simple calculation shows that, for every $i \in \mathbf{N}$ and $1 \leq j \leq 2^{i}$, each interval $I_{i, j}$ has length

$$
\frac{2^{i}+1}{2^{2 i+1}} \in\left(2^{-i-1}, 2^{-i}\right]
$$

Thus $C_{i}$, and hence $E$ is well-defined. Moreover, $E$ has positive $\mathcal{H}^{1}$-measure; note that at step $i, i \geq 1$ there are $2^{i}$ intervals removed with total length $2^{-i-1}$.

We now construct a sequence of simple curves $\gamma_{i}$ based on the construction of $E$. Again we proceed inductively according to the index $i$. For $i \in \mathbf{N}$ and $1 \leq j \leq 2^{i}$, denote by $I_{i, j}^{\prime} \subset I_{i, j}$ the interval removed from $I_{i, j}$ in the construction of $E$. Let $\gamma_{0}$ be the interval $[0,1]$. When $\gamma_{i-1}, i \geq 1$ has been defined, we replace every open interval $I_{i, j}^{\prime}, 1 \leq j \leq 2^{i}$, contained in $\gamma_{i-1}$, by a curve

$$
\gamma_{i, j}=\partial\left(I_{i, j}^{\prime} \times\left[0,2^{-i}\right]\right) \backslash\left(I_{i, j}^{\prime} \times\{0\}\right)
$$

consisting of three line segments, where $\times$ means the Cartesian product. We then obtain $\gamma_{i}$. See Figure 1.

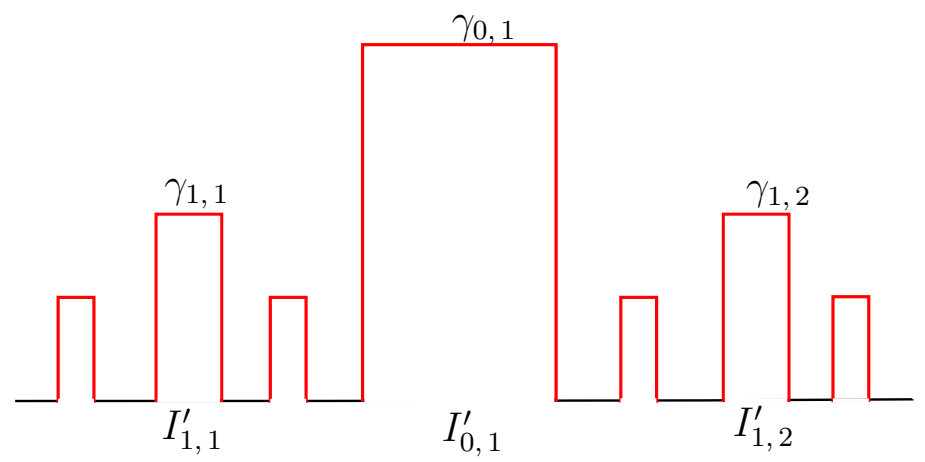

Figure 1. The curve $\gamma_{2}$ is shown in the figure. In the previous steps the intervals $I_{0,1}^{\prime}, I_{1,1}^{\prime}, I_{1,2}^{\prime}$ were replaced by curves $\gamma_{0,1}, \gamma_{1,1}, \gamma_{1,2}$, and in the current step four more intervals are replaced.

Since $\left\{\gamma_{i}\right\}$ (under suitable parameterizations) is a Cauchy sequence of curves in the plane with respect to the supremum distance, the limit $\gamma$ exits and is a curve. Moreover, $\gamma$ is simple.

For fixed $n \in \mathbf{N}$, there are $2^{n+1}-1$ curves $\gamma_{i, j}$ intersecting $\mathbf{R} \times\left(2^{-n-1}, 2^{-n}\right]$. Indeed, if $\gamma_{i, j} \cap\left(\mathbf{R} \times\left(2^{-n-1}, 2^{-n}\right]\right) \neq \emptyset$, then $i \leq n$. The distance between any two of these curves is strictly larger than $2^{-n-1}$ by $(2.1)$.

We next construct a sequence of new curves $\Gamma_{n}$ according to the index $n$. First of all define $\Gamma_{0}=\gamma$. When $\Gamma_{n-1}, n \geq 1$ has been defined, we modify the segments in

$$
\gamma_{i, j} \cap\left(\mathbf{R} \times\left(2^{-n-1}, 2^{-n}\right]\right), \quad 0 \leq i \leq n,
$$

to obtain $\Gamma_{n}$. Recall that $\gamma_{i, j}$ replaces the interval $I_{i, j}^{\prime}$ in the construction of $\gamma_{i}$. Denote by $a_{i, j}$ and $b_{i, j}$ the end points of $I_{i, j}^{\prime}$ with $a_{i, j}<b_{i, j}$. Then for every $1 \leq i \leq n$,

$$
\gamma_{i, j} \cap\left(\mathbf{R} \times\left(2^{-n-1}, 2^{-n}\right]\right)=\left(\left\{a_{i, j}\right\} \times\left(2^{-n-1}, 2^{-n}\right]\right) \cup\left(\left\{b_{i, j}\right\} \times\left(2^{-n-1}, 2^{-n}\right]\right)
$$


and each $1 \leq k \leq 2^{n}-1$, we replace each segment

$$
\left\{a_{i, j}\right\} \times\left[2^{-n-1}+(4 k) 2^{-2 n-3}, 2^{-n-1}+(4 k+1) 2^{-2 n-3}\right]
$$

by

$$
\begin{aligned}
& A_{i, j}^{n, k}:=\partial\left(\left[a_{i, j}-2^{-n-1}, a_{i, j}\right] \times\left[2^{-n-1}+(4 k) 2^{-2 n-3}, 2^{-n-1}+(4 k+1) 2^{-2 n-3}\right]\right) \\
& \backslash\left\{a_{i, j}\right\} \times\left[2^{-n-1}+(4 k) 2^{-2 n-3}, 2^{-n-1}+(4 k+1) 2^{-2 n-3}\right],
\end{aligned}
$$

and

$$
\left\{b_{i, j}\right\} \times\left[2^{-n-1}+(4 k+2) 2^{-2 n-3}, 2^{-n-1}+(4 k+3) 2^{-2 n-3}\right]
$$

by

$$
\begin{aligned}
& B_{i, j}^{n, k}:=\partial\left(\left[b_{i, j}, b_{i, j}+2^{-n-1}\right] \times\left[2^{-n-1}+(4 k+2) 2^{-2 n-3}, 2^{-n-1}+(4 k+3) 2^{-2 n-3}\right]\right) \\
& \backslash\left\{b_{i, j}\right\} \times\left[2^{-n-1}+(4 k+2) 2^{-2 n-3}, 2^{-n-1}+(4 k+3) 2^{-2 n-3}\right] .
\end{aligned}
$$

This gives us the new curve $\Gamma_{n}$. See Figure 2 .

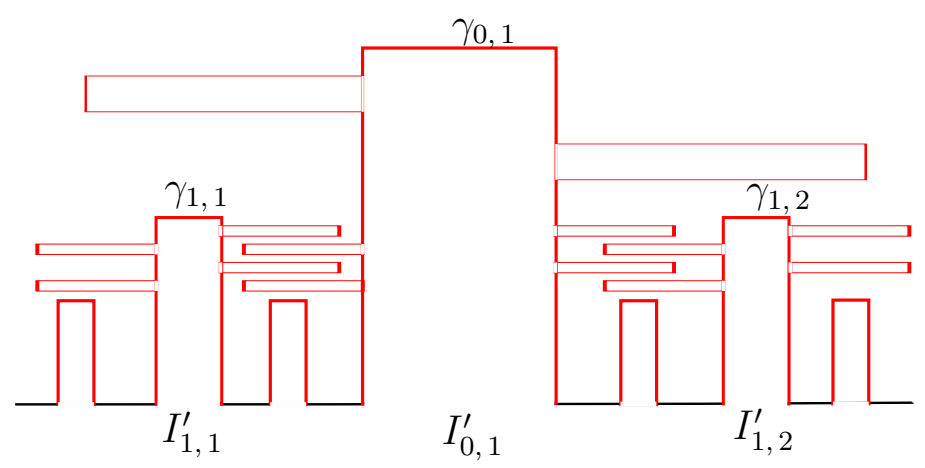

Figure 2. The curve $\Gamma_{2}$ is shown in the figure, with the replacement of certain segments contained in $\Gamma_{1}$ by parts of boundaries of some rectangles, receptively.

Again since $\left\{\Gamma_{n}\right\}$ (under suitable parameterizations) is a Cauchy sequence of curves in the plane with respect to the supremum distance, we conclude that $\Gamma_{n}$ converges uniformly to some curve $\Gamma_{\infty}$ as $n \rightarrow \infty$. Moreover, according to our construction $\Gamma_{\infty}$ is simple. Define

$$
\Gamma=\Gamma_{\infty} \cup(\partial([0,1] \times[-1,0]) \backslash[0,1] \times\{0\}) .
$$

Since $\Gamma_{\infty}$ is simple, then also $\Gamma$ is simple, and hence Jordan as it is closed. We denote by $\Omega$ the bounded component of $\mathbf{C} \backslash \Gamma$.

Notice that $\partial \Omega$ is a countable union of rectifiable curves, even though it does not have finite length. Since the Hausdorff dimension of a countable union of sets is the supremum of the Hausdorff dimensions of the sets, see e.g. [11, Page 81, Section 5.9], we conclude that $\partial \Omega$ is a set of Hausdorff dimension 1.

Recall the Cantor set $E$. Now let us check that the Jordan domain $\Omega$ satisfies the two properties (i) and (ii). We remark that, in our construction, for any point $x$ in $\Omega$, we have

$$
\operatorname{dist}_{\Omega}(x, E)<\infty .
$$

Before showing (i), we note that, property (i) is stated with respect to all conformal maps. However, since two such Riemann maps differ from each other by a Möbius transform on the unit disk, we may assume that $\varphi(0)$ is the center of the square $[0,1] \times[-1,0]$.

Recall that the harmonic measure in the unit disk is defined via the Poisson kernel, and then in any Jordan domain via the (extended) Riemann mapping. For 
$E \subset \partial \Omega$, we use $\omega\left(x_{0}, E, \Omega\right)$ to designate the harmonic measure of $E$ at $x_{0}$ in $\Omega$. It is known that $\omega\left(x_{0}, E, \Omega\right)=u\left(x_{0}\right)$ where $u$ is the (unique) harmonic function in $\Omega$ whose boundary value is the characteristic function of $E$ on $\partial \Omega$. We refer to [4] for more details.

Lemma 2.1. The Jordan domain $\Omega$ constructed above satisfies properties (i) and (ii).

Proof. Towards (i), we first observe that

$$
\omega(\varphi(0), E, \Omega) \geq \omega(\varphi(0), E, Q)>0,
$$

where $Q$ is the open square $(0,1) \times(-1,0)$. Indeed, the first estimate comes from the comparison principle of harmonic measures, while the second inequality follows from F. and M. Riesz theorem since its 1-Hausdorff measure is strickly positive.

By the conformal invariance of harmonic measure we have

$$
\omega\left(0, \varphi^{-1}(E), \mathbf{D}\right)>0 .
$$

According to the definition of harmonic measures in the unit disk, we conclude (i).

To show (ii), note that in our construction, any curve in the unbounded component of $\mathbf{R}^{2} \backslash \Gamma$ towards $E \backslash\{(0,0),(1,0)\}$ has length at least $\frac{1}{2}$ in the region $\mathbf{R} \times\left(2^{-n-1}, 2^{-n}\right]$ for $n$ large enough; the curve has to oscillate $2^{n}$ times and each time it goes at least $2^{-n-1}$. This implies that any curve in the unbounded component of $\mathbf{R}^{2} \backslash \Gamma$ towards $E \backslash\{(0,0),(1,0)\}$ has infinite length. Property (ii) is complete.

2.2. Schoenflies solution of conformal boundary value $\phi: \partial \Omega \rightarrow \mathrm{S}^{\mathbf{1}}$. Let $\phi: \Omega \rightarrow \mathbf{D}$ be a conformal map giving the conformal boundary value via Carathéodory's theorem. By composing with a suitable Möbius map, we may assume that $\phi\left(z_{0}\right)=0$, where $z_{0}$ is the center of open square $Q=(0,1) \times(-1,0)$; in the general case the constants below will further depending on the Möbius transform. We show that any homeomorphic extension of $\phi$ is not in $W_{\text {loc }}^{1,1}$.

Towards this, recall that in the construction of $\Gamma=\partial \Omega$ we attached "arms" $A_{i, j}^{n, k}$ and $B_{i, j}^{n, k}$ to every curve $\gamma_{i, j}$. We first claim that, there exists an absolute constant $c>0$ such that, for $n \geq 3$,

$$
\operatorname{dist}\left(\phi\left(A_{i, j}^{n, k}\right), \phi\left(B_{i^{\prime}, j^{\prime}}^{n, k^{\prime}}\right)\right) \geq c 2^{-n}
$$

whenever $I_{i, j}^{\prime}, I_{i^{\prime}, j^{\prime}}^{\prime} \subset\left[\frac{5}{32}, \frac{27}{32}\right]$ and either $i \neq i^{\prime}$ or $j \neq j^{\prime}$.

Indeed, let us fix $A_{i, j}^{n, k}$ and $B_{i^{\prime}, j^{\prime}}^{n, k^{\prime}}$. According to our construction, there exists an interval $J \in\left\{I_{n+1, j}\right\}_{j=1}^{2^{n+1}}$ such that $J \subset\left[\frac{5}{32}, \frac{27}{32}\right]$ is between $I_{i, j}^{\prime}$ and $I_{i^{\prime}, j^{\prime}}^{\prime}$. Since $\phi: \partial \Omega \rightarrow \mathbf{S}^{1}$ is a homeomorphism, by the construction of $\partial \Omega$ and the geometry of the unit circle, we have that

$$
\operatorname{dist}\left(\phi\left(A_{i, j}^{n, k}\right), \phi\left(B_{i^{\prime}, j^{\prime}}^{n, k^{\prime}}\right)\right) \geq c_{1} \mathcal{H}^{1}(\phi(J \cap E))
$$

for some absolute constant $c_{1}$. Therefore it suffices to show that $\mathcal{H}^{1}(\phi(J \cap E)) \geq c_{2} 2^{-n}$ for some absolute constant $c_{2}$.

Again by the invariance of harmonic measure under conformal map and the comparison principle of harmonic measures,

$$
\omega(0, \phi(J \cap E), \mathbf{D})=\omega\left(z_{0}, J \cap E, \Omega\right) \geq \omega\left(z_{0}, J \cap E, Q\right) .
$$


According to Schwarz-Christoffel formula [12, Chapter 3.1], since $J \subset\left[\frac{5}{32}, \frac{27}{32}\right]$ is away from the corner of $Q$, we have

$$
\omega\left(z_{0}, J, Q\right) \geq c_{3} 2^{-n}
$$

for some absolute constant $c_{3}$; note that the length of $J$ is $2^{-n-2}+2^{-1} 4^{-n-1}$, and $E$ is a self-similar fat Cantor set. Therefore, we conclude (2.3) via the Poisson formula in the unit disk.

Let $\Phi$ be any Schoenflies solution of the conformal boundary value $\phi$. By (2.3), the image under $\phi$ of any vertical segment joining "neighboring arms" $A_{i, j}^{n, k}$ and $B_{i^{\prime}, j^{\prime}}^{n, k}$ in the exterior of $\Omega$ has length at least $c 2^{-n}$. Moreover, when $n \geq 3$, the intersection of the projections on the real axis of the "neighboring arms" $A_{i, j}^{n, k}$ and $B_{i^{\prime}, j^{\prime}}^{n, k}$ is an interval with length not less than $2^{-n-2}$, and there are at least $4^{n}$ pairs of those "neighboring arms" contained in $\left[\frac{5}{32}, \frac{27}{32}\right] \times\left[2^{-n-1}, 2^{-n}\right]$ up to a multiplicative constant. Therefore by Fubini's theorem we conclude

$$
\int_{\left[\frac{5}{32}, \frac{27}{32}\right] \times\left[2^{-n-1}, 2^{-n}\right]}|D \Phi| d x \geq c^{\prime} 2^{-n} 2^{-n-2} 4^{n} \geq c^{\prime} 2^{-2}
$$

for some absolute constant $c^{\prime}$. Therefore, in any Euclidean neighborhood of $E \cap$ $\left[\frac{5}{32}, \frac{27}{32}\right]$ the $W_{\text {loc }}^{1,1}$-energy of $\Phi$ is infinite, and a similar argument shows that $\Phi \notin$ $B V_{\text {loc }}$. This concludes the second part of Theorem 1.2.

\section{References}

[1] Astala, K.: Area distortion of quasiconformal mappings. - Acta Math. 173:1, 1994, 37-60.

[2] Astala, K., T. Iwaniec, G. J. Martin, and J. Onninen: Extremal mappings of finite distortion. - Proc. London Math. Soc. 91:3, 2005, 655-702.

[3] Bell, S. R., and S. G. Krantz: Smoothness to the boundary of conformal maps. - Rocky Mountain J. Math. 17:1, 1987, 23-40.

[4] Garnett, J. B., and D. E. Marshall: Harmonic measure. - New Math. Monogr. 2, Cambridge Univ. Press, Cambridge, 2005.

[5] Genring, F. W.: The $L^{p}$-integrability of the partial derivatives of a quasiconformal mapping. - Acta Math. 130, 1973, 265-277.

[6] Iwaniec, T., G. Martin, and C. Sbordone: $L^{p}$-integrability \& weak type $L^{2}$-estimates for the gradient of harmonic mappings of D. - Discrete Contin. Dyn. Syst. Ser. B 11:1, 2009, $145-152$.

[7] Koskela, P.: Lectures on quasiconformal and quasisymmetric mappings. - University of Jyväskylä.

[8] Koskela, P., P. Pankka, and Y. R.-Y. Zhang: Generalized uniform domains. - In progress.

[9] Koskela, P., Z. WAng, and H. XU: Controlled diffeomorphic extension of homeomorphisms. - arXiv:1805.02906.

[10] Koski, A., and J. Onninen: Sobolev homeomorphic extensions. - arXiv:1812.02085.

[11] Mattila, P.: Geometry of sets and measures in Euclidean spaces: fractals and rectifiability. - Cambridge Univ. Press, 1995.

[12] Pommerenke, Ch.: Boundary behaviour of conformal maps. - Grundlehren Math. Wiss. 299, Springer-Verlag, Berlin, 1992.

[13] Verchota, G. C.: Harmonic homeomorphisms of the closed disc to itself need be in $W^{1, p}$, $p<2$, but not $W^{1,2}$. - Proc. Amer. Math. Soc. 135:3, 2007, 891-894. 